\title{
Hybrid Intelligent Algorithm for Flexible Job-Shop Scheduling Problem under Uncertainty
}

\author{
Guojun Zhang, Haiping Zhu and Chaoyong Zhang \\ School of Mechanical Science \& Engineering, \\ Huazhong University of Science \& Technology \\ Wuhan \\ P.R. China
}

\section{Introduction}

Production scheduling plays an important role in improving efficiency and reducing cost. One of its core technologies is the establishment of an effective scheduling model and its corresponding optimization algorithms. However, most researches focus on scheduling optimization in static environment, less concern of the uncertainty and complexity in the real job-shop. It must be different from the exact solution if some situations are ignored or not considered in scheduling problem such as changing processing time, uncertain capability of storage, possibility of human decision, unpredicted accident and so on.

Inchoate research on FJSP (Flexible Job-Shop Scheduling Problem) concentrated on the simple application of integer programming and simulation, which can hardly be used to solve the complex scheduling problem. With the development of related fields and theory of optimization, a great many methods and techniques have been adopted into JSP. Operational research predigests the JSP into a mathematical programming model, using branch-and-bound method and dynamic programming algorithm to realize optimization or approximate optimization. However, OR only suits to simple scheduling problem (Philips et al., 1987). Based on theory of control, Gershwin and his fellows expatiate comprehensively the adoption of theory of control in manufacturing system. Limited to the capability of modeling, a lot of predigestion to the environment is a must; the practice to get the optimum solution expands with a exponential characteristic (Juanqi, 1998). AI (Artificial Intelligence) is a combination of all the methods for JSP, which aim at enhancing the intelligence of scheduling method. It can smooth the disadvantages of mathematical programming and simulation. Based on the system status and deterministic objective of optimization, effective heuristic research and concurrent fuzzy reasoning are conducted to choose the optimum solution and support online decision. Nevertheless, AI is weak in adapting to new environment, and there are 3 main limitations of this method: low speed of operation, insensitive to asynchronisic event in the environment, the system can't be universally adopted (Juanqi, 1998). In a job-shop DEDS (Discrete Event Dynamic System), the JSP can be 
solved by its parsing model and method, such as Petri net. The Petri net can precisely reflect the characteristics of discrete and stochastic etc. in job-shop scheduling (Hongsen, 1994). But it's hard to present a model when the principle and methodology are complex. The complexity of manufacturing system makes it hard to express and analyze by a precise analytical model. While simulation can provide an ideal model for quantitative evaluation to guide the scheduling (Law, 1986). Whereas, the experimental characteristic make it hard to figure out the general rule, and the value and credibility of the result have much to do with the simulation model, simulation method and the data input. Uncertain theory has also been adopted into the scheduling problem for its stochastic and fuzzy characteristics (Zhaoqiang \& Yiren, 2002). Just as AI, it needs a long development cycle time as well as abundant experience etc. Soft computing is also widely adopted into scheduling, such as genetic algorithm (Croee \& Tadei, 1995), Tabu searching (Taillard, 1990), simulated annealing (Van Laarhoven, 1992), neural network (Foo \& Takefuji, 1998), particle swarm optimization etc. Based on the aforementioned methodologies, we can find that each method has its own limit, so researchers start to combine the approximate algorithms to solve scheduling problem. This paper proposed a compounding method which syncretises simulation and soft computing to solve FJSP (flexible job-shop scheduling problem) under uncertain environment.

As the execution layer between the ERP/MRPII for planning and job-shop layer for operation and control, MES (Manufacturing Execution System) focuses on how to optimize the job-shop scheduling (Shuxia, 2004). The essence of scheduling is a decision making constrained by multi-objectives and multi-priorities. The multi-objectives here focuses on the combination of less production time, less total cost and equilibrium of production ability while the multi-constraints focuses on the constraints of process route, machines and delivery time of order in which the priorities differ on different types of work pieces.

Information is the basis of decision making. The scheduling models in previous research are proposed with all the information deterministically given, for example, taking the process time of the work piece on specific machine as deterministic information without considering the priority. Actually, the stochastic information floods the real job-shop, which makes getting the satisfied scheduling a difficult job. In recent years, job-shop scheduling in uncertain environment gradually arouses the attention of researchers, such as study on scheduling problem with fuzzy characteristic. However, in discrete event dynamic system, more parameters are suited to be described by stochastic variable rather than fuzzy variable, such as the process time of work piece, the frequency of machine fault and the interval of order, which can be thought to obey an exponential distribution (Peigen, 1998), so it is of great significance to study scheduling under stochastic constraints.

In this paper, describing the uncertain information in the real job-shops with several stochastic variables, a stochastic multi-objectives and multi-priorities programming model for Flexible job-shop scheduling is proposed, in which Time, Cost and Equilibrium serve as the three basic objectives for scheduling. The credibility of the delivery time of different types of work pieces serve as the scheduling constraints. In order to obtain the approximate optimum solution, a hybrid intelligent algorithm which combines Stochastic Simulation (SS), Neural Network (NN) with Genetic Algorithm (GA) is proposed. The feasibility of the 
model and its corresponding hybrid intelligent algorithm is validated through some instances.

\section{The stochastic programming model with chance-constraint}

Generally, job-shop problem is depicted by a non-linear programming model. If certain stochastic factors being taken into account, the programming model would differ from the classical model. The concept of chance-constraint and stochastic programming proposed by Liu (Baoding et al., 2003) is depicted as follows:

Assume that $X$ is a decision-making vector; $E$ is a stochastic vector with deterministic distribution. There are $r$ objective functions decided by $X$ and $E: f_{i}(X, E), 1 \leq i \leq r$, constrained to $p$ constrained function: $g_{j}(X, E), 1 \leq j \leq p$. The objective functions and constrained functions are stochastic, so they can be depicted only by the credibility as follows: $P\left\{g_{j}(X, E) \leq 0\right\} \geq \alpha_{j}$, $1 \leq j \leq p, P$ represent the probability of the stochastic event $g_{j}(X, E) \leq 0, \alpha_{j}$ is the credibility. If the objective of programming is to minimize $f_{i}(X, E), 1 \leq i \leq r$, choose $\beta_{i}$ as the credibility, the objective function would be:

$$
\left\{\begin{array}{l}
\min \bar{f}_{i} \\
\text { s.t. } \quad P\left\{f_{i}(X, E) \leq \bar{f}_{i}\right\} \geq \beta_{i}
\end{array}, 1 \leq i \leq r\right.
$$

Above all, the stochastic programming model with multi-chance-constraints and multi objectives is:

$$
\left\{\begin{array}{l}
\min \left[\bar{f}_{1}, \bar{f}_{2}, \ldots, \bar{f}_{r}\right] \\
\text { s.t. } \\
\quad P\left\{f_{i}(X, E) \leq \bar{f}_{i}\right\} \geq \beta_{i}, 1 \leq i \leq r \\
\quad P\left\{g_{j}(X, E) \leq 0\right\} \geq \alpha_{j}, 1 \leq j \leq p
\end{array}\right.
$$

$\left[\bar{f}_{1}, \bar{f}_{2}, \ldots, \bar{f}_{r}\right]$ stands for the weighted sum of the r variables.

\section{Multi-objectives and multi-priorities flexible job-shop scheduling strategy}

In the Flexible job-shop with several pieces of machines, after receiving the production order assigned by the planning section, production process would be arranged and optimized by reasonable scheduling. The scheduling parameter can be depicted by the mathematical format: There are $\mathrm{m}$ pieces of machine available: $\left\{R_{1}, R_{2}, \ldots . R_{m}\right\}$

There are $n$ work pieces' task included in the order: $\left\{T_{1}, T_{2}, \ldots T_{m}\right\} T_{i}(1 \leq i \leq n)$ is a operation sequence decided by the process route programming, which includes $K_{i}\left(1 \leq K_{i} \leq m\right)$ operations and recorded as follows: $T_{i}=\left\{O P_{i 1}, O P_{i 2}, \ldots, O P_{i K}\right\}$, each operation $O P_{i k}$ can be processed on several machines, and operation on each machine $R_{j}$ would take you certain time and cost, time function be depicted as $\operatorname{et}(O P, R)$, cost function be depicted as $e c(O P, R)$. In the uncertain informative environment, when operation $O P_{i k}\left(1 \leq i \leq n, 1 \leq k \leq K_{i}\right)$ is being processed on machine $R_{j}(1 \leq j \leq m)$, et $\left(O P_{i k}, R_{j}\right)$ and $e c\left(O P_{i k}, R_{j}\right)$ are two stochastic constants with deterministic distribution, else $e t\left(O P_{i k}, R_{j}\right)=e c\left(O P_{i k}, R_{j}\right) \equiv 0$. 


\subsection{Work piece priority and chance constraint}

On the premise of following the demand of process route, MES would assign appropriate machine for each operation of each work piece, making sure that the delivery time of order can be met. That is to say, the delivery time is a key constraint in scheduling. As the production order is formed by a consideration of customer order, enterprise planning and operation. The tasks included in the order can be divided into many priorities for many factors such as importance of customer, quantity of order, and the degree of emergency of the requirement. For example, if we divide the work pieces into two group, in one of which the delivery time should be precisely met (called critical work piece) while there can be a flexible space in the other group of work pieces (called ordinary work piece). On this condition, the job-shop resources should satisfy the needs of critical work pieces on first hand to meet the delivery time. The remained resources can then be assigned to the ordinary work pieces. Literature 4 adopted by-directional scheduling strategy, for the critical work piece, reverse order scheduling is adopted, while for the ordinary work piece, sequential scheduling is adopted. However, for uncertain environment in which processing time is stochastic, this strategy for scheduling will bring out many problems. That means the result of the scheduling can hardly meet the delivery time.

In real job-shop, the processing time of work piece on each machine is a stochastic variable obeying certain distribution (exponential distribution in general), that leads to the stochastic characteristic of the production cycle of work piece. We can describe the constraint of delivery time as a chance constraint. The priority of the work pieces can be divided into $\mathrm{r}$ subclasses by a high-low order (the element in the same subclass share the same priority): $S_{1}, S_{2}, \ldots, S_{r}$, each subclass has the corresponding credibility of meeting delivery time: $\alpha_{1}, \alpha_{2}, \ldots \alpha_{r}\left(\alpha_{1}>\alpha_{2}>\ldots>\alpha_{r}\right)$. The delivery time of each task is deterministic as: $d t_{1}, d t_{2}, \ldots, d t_{n}$ the real production cycle time of each task(including the total processing time and total waiting time) can be calculated and depicted as $\mathrm{pt}_{1}, \mathrm{pt}_{2}, \ldots, \mathrm{pt}_{\mathrm{n}}$. The delivery constraint described by chance constraint is:

$$
\forall T_{i} \in S_{j}, P\left\{p t_{i}-d t_{i} \leq 0\right\} \geq \alpha_{j}, 1 \leq i \leq n, 1 \leq j \leq r
$$

There would be a queue waiting when several work pieces are assigned to the same machine, the sequence of processing should be determined by the principle of scheduling. Generally, FIFS (first in first serviced) principle is adopted. Towards different priorities, serving the task with higher priority on first hand is more reasonable to actual demand. At this moment, if there is a queue on a machine waiting for processing while a piece is being processed, after it is processed, the piece in queue with highest priority should be chosen to be processed. The piece of longer waiting time should be processed first within the same priority.

\subsection{Proposing a multi-objectives function}

Shorter production time and lower cost is two basic objectives. For an order to be scheduled, with the complexity of process route of each component differing from others, we can aim at minimize the total production cycle time of the task for all work pieces. $\eta$ as the credibility, the time objective function can be depicted by chance constraint as follows: 


$$
\left\{\begin{array}{l}
\min \overline{p t} \\
\text { s.t. } P\left\{\sum_{i=1}^{n} p t_{i} \leq \overline{p t}\right\} \geq \eta
\end{array}\right.
$$

Meanwhile, we can aim at minimizing the total production cost of the task for all work pieces, taking $\gamma$ as credibility. The cost objective function can be depicted by chance constraint as follows:

$$
\left\{\begin{array}{l}
\min \overline{e c} \\
\text { s.t. } P\left\{\sum_{i=1}^{n} \sum_{k=1}^{K_{i}} e c\left(O P_{i k}, R_{j}\right) \leq \overline{e c}\right\} \geq \gamma
\end{array}\right.
$$

Another objective of scheduling is the equilibrium of the schedule, which has long been ignored. According to a schedule, if the average load of machine is floating dramatically, we call it an unbalanced scheduling. The disequilibrium of scheduling would cause a loss in coping with the emergency. We introduce the standard deviation in statistics to quantify the equilibrium of scheduling, dividing the whole production cycle time into several isometric time segment, figure out the total working time of the machine in each time segment, than calculate the standard deviation between this total working time and average working time. The smaller the standard deviation, the better the equilibrium of the schedule is.

Assume that the production cycle time is $O T$, obviously, $O T=\max _{1 \leq i \leq n} p t_{i}$, divide OT into $N$ isometric time segments, in each of which the total processing time of all the machines is $\Delta R T_{s}(1 \leq s \leq N)$, the standard deviation is $D^{2}=\sum_{s=1}^{N}\left(\Delta R T_{s}-\frac{1}{N} \sum_{s=1}^{N} \Delta R T_{s}\right)^{2}$, with credibility $v$, the objective of equilibrium of schedule is depicted as:

$$
\left\{\begin{array}{l}
\min \overline{r t} \\
\text { s.t. } P\left\{\sum_{s=1}^{N}\left(\Delta R T_{s}-\frac{1}{N} \sum_{s=1}^{N} \Delta R T_{s}\right)^{2} \leq \overline{r t}\right\} \geq v
\end{array}\right.
$$

\subsection{Stochastic programming model of job-shop scheduling with multi-objectives and multi-priorities}

The process route of each work piece and the machines available for each operation are known according to (1) (2) (3) (4), the machines available to operation $O P_{i k}$ is $A R_{i k}=\left\{R_{i k}^{1}, R_{i k}^{2}, \ldots, R_{i k}^{H_{i k}}\right\}$, including $H_{i k}$ elements, the principle for scheduling of task waiting in queue is Higher Priority First Served. The stochastic programming model of jobshop scheduling in uncertain environment is proposed as follows: 


$$
\begin{cases}\min [\overline{p t}, \overline{e c}, \overline{r t}] \\ \text { s.t. } \quad P\left\{\sum_{i=1}^{n} p t_{i} \leq \overline{p t}\right\} \geq \eta \\ & P\left\{\sum_{i=1}^{n} \sum_{k=1}^{K_{i}} e c\left(O P_{i k}, R_{j}\right) \leq \overline{e c}\right\} \geq \gamma \\ & P\left\{\sum_{s=1}^{N}\left(\Delta R T_{s}-\frac{1}{N} \sum_{s=1}^{N} \Delta R T_{s}\right)^{2} \leq \overline{r t}\right\} \geq v \\ & P\left\{p t_{i}-d t_{i} \leq 0\right\} \geq \alpha_{j}, 1 \leq i \leq n, 1 \leq j \leq r, T_{i} \in S_{j}\end{cases}
$$

\section{The design and implementation of hybrid intelligent algorithm}

\subsection{Basic procedures of hybrid intelligent algorithm}

Model (5) is too complex to be solved by traditional algorithm, so we adopt the hybrid intelligence which syncretizes stochastic simulation, neural network, genetic algorithm and other critical techniques. The algorithm includes 3 major steps:

Step 1. based on the quantity and capability of machine in job-shop, as well as the distribution characteristics of stochastic variables as time and cost etc. Present a simulation model; collect quantities of data samples by simulating.

Step 2. Present the three-layer feed-forward neural networks, use the samples acquired in simulation to weight-train the neural network to approach the stochastic functions in model(5).

Step 3. apply the genetic algorithm to solve the optimization problem, including definition of rules for chromosome coding/encoding, initialization and selection of population, the calculation and evaluation of individual fitness, crossover and mutation etc. The calculation of individual fitness will utilize the trained neural network in step2.

\subsection{Stochastic simulations}

Stochastic simulations( Monte Carlo simulation) is one of the stochastic system modeling techniques focuses on sample test, the sample of the stochastic variables is based on their probabilistic distribution. There are a lot of complex calculations for probabilities of stochastic variables in model (5), as for the complexity of multi-priorities scheduling, even a schedule sequence is determined, the stochastic variables such as the completion period of each work piece can not be expressed by a explicit. So the stochastic simulation should be integrated with simulation analysis to get the approximate sample data.

According to experience, we can assume the operation time et $\left(O P_{i k}, R_{j}\right)$ comply with the exponential distribution with $\lambda_{i k j}$ as its exponent, depicted as:

$$
\text { et }\left(O P_{i k}, R_{j}\right) \sim \exp \left(\lambda_{i k j}\right) \text {. }
$$

The cost of processing is influenced by many uncertain factors, ec $(O P i k, R j)$ can be assumed to comply with normal distribution depicted as:

$$
\mathrm{ec}\left(O P_{i k}, R_{j}\right) \sim \mathrm{N}\left(\mu_{i k j}, \sigma_{i k j}^{2}\right) .
$$


Given a schedule sequence (the concrete machine for each operation) and a group of deterministic sample values for all stochastic variables, which work pieces are being processed is available on theory. Therefore, to present stochastic simulation model on platform of SIMUL8, then run simulation and statistically analyze the critical data acquired, including:

1. Production time of each work piece $\mathrm{pt}_{\mathrm{i}}$

2. The processing cost of each work piece $\sum_{k=1}^{K_{i}} e c\left(O P_{i k}, R_{j}\right)$

3. The total working time of all devices in each time segment ( $\mathrm{N}$ segment in all) is $\Delta R T_{s}$

\subsection{Neural network approach to the stochastic functions}

Take the schedule sequence as Vector $Y$, the four constraints in model (5) can be depicted by $Y^{\prime}$ s stochastic function, definition as the following $n+3$ functions:

$$
\left\{\begin{array}{l}
U_{1}(Y)=\min \left\{\overline{p t} \mid P\left\{\sum_{i=1}^{n} p t_{i} \leq \overline{p t}\right\} \geq \eta\right\} \\
U_{2}(Y)=\min \left\{\overline{e c} \mid P\left\{\sum_{i=1}^{n} \sum_{k=1}^{K_{i}} e c\left(O P_{i k}, R_{j}\right) \leq \overline{e c}\right\} \geq \gamma\right\} \\
U_{3}(Y)=\min \left\{\overline{r t} \mid P\left\{\sum_{s=1}^{N}\left(\Delta R T_{s}-\frac{1}{N} \sum_{s=1}^{N} \Delta R T_{s}\right)^{2} \leq \overline{r t}\right\} \geq v\right\} \\
U_{4}^{(i)}(Y)=P\left\{p t_{i}-d t_{i} \leq 0\right\}, 1 \leq i \leq n
\end{array}\right.
$$

The stochastic simulation provides abundant data samples, which are used to train a neural network to approach the $n+3$ uncertain functions in (6). The neural network is three-layer feed-forward with $\sum K_{i}$ input neurons (data input is $Y$ ), 18 hidden layer neurons and $n+3$ output neurons (datẫ output are U1(Y), U2(Y), U3(Y), $U_{4}^{(i)}(Y)$ ).

\subsection{Genetic algorithm of multi-objectives optimization}

Coding, encoding of chromosome and initialization of population. Besides being simple, the principle for coding of chromosome should also assure any of the chromosomes can get a permissive schedule sequence by encoding. Permissive means meeting the requirements of constraints of process route and process machine. Here designed a coding method for positive integer:

The total length of coding is, each bit represents an operation: the first bit represents the first operation of work piece 1 , the second bit represents the second operation of work piece $1, \ldots$, the $K_{1+1}$ represents the first operation of work piece 2, the rest may be deduced by analogy. If bit $\mathrm{j}$ represents operation $\mathrm{k}$ of work piece of $\mathrm{I}$, the range of value for bit $\mathrm{j}$ is $\left[1, H_{i k}\right], H_{i k}$ represents the number of machine available for operation $\mathrm{k}$ of work piece i. Looking on the coding principle, once given the collection of machine for each operation and the accurate process time, each chromosome can be precisely mapped into a schedule sequence, meanwhile the process details (when and where) of each operation can be calculated which is solely determined. Also, during the initialization of population, once length bits stochastic positive integers are created with value-range of each bit in $\left[1, H_{i k}\right]$, the coding of chromosome is permissive. 
The calculation and evaluation of fitness. Individual fitness can be calculated using the trained neural network, if we take $\sum^{n} K_{i}$ bits coding of random chromosomes as the input of neural network, $\mathrm{n}+3$ output woulfd be acquired. Contraposing data output first prove whether $U_{4}^{(i)}(Y) \geq \alpha_{j}, T_{i} \in S_{j}$ is right, if not, the delivery time can not be satisfied which means the schedule is not donable. The fitness of chromosome is 0 , the corresponding individual being eliminated. Else when $U_{4}^{(i)}(Y) \geq \alpha_{j}, T_{i} \in S_{j}$ proves to be true, then $U 1(Y)$, $U 2(Y)$ and $U 3(Y)$ can figure out $\overline{p t}, \overline{e c}, \overline{r t}$, giving the weight of the three objectives(time, cost and equilibrium) $w_{1}, w_{2}, w_{3}$ on preferential relationship, then calculate $w_{1} \overline{p t}+w_{2} \overline{e c}+w_{3} \overline{r t}$, the smaller the value, the higher the individual fitness is.

Operator of cross and mutation. The cross between two parents is operated as follows: exchange the codes presenting the same operation sequence in two chromosomes, and then we will get two sons.

The mutation process is as fig. 2, bit $j$ of parent's chromosome mutates stochastically by certain probability. Notes: if bit $\mathrm{j}$ stands for the operation $k$ of work piece $\mathrm{I}$, the value range of bit $j$ after mutation is $\left[1, H_{i k}\right]$.

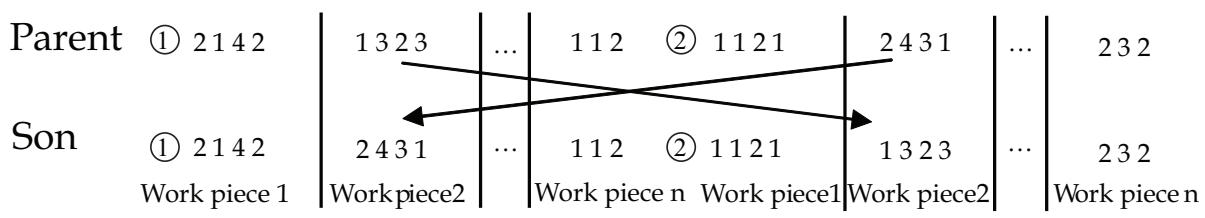

Fig. 1. Process of crossing

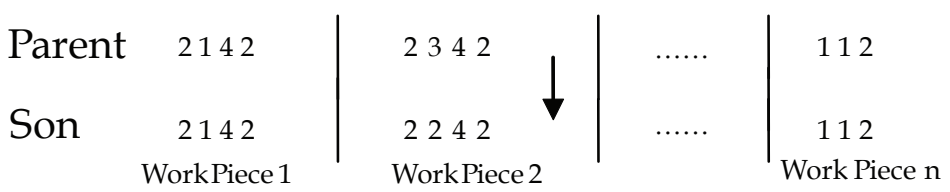

Fig. 2. Process map of mutation

\section{Case study}

We apply our study to a car manufacturing enterprise. The mold job-shop accepts the orders for manufacturing of molds, which include orders from both enterprise itself and other company. The manufacturing resources in the job-shop is enough with all necessary machine for mold processing, which include lathe, milling machine, grinding machine, numeral control machine, process center, electric discharge machine etc.(the serial number of machines are $R_{1}, R_{2}, \ldots, R_{35}$ ). Table 1 shows a piece of production order, which includes 3 work pieces waiting for process with delivery time of $270,300,320$ respectively. The delivery time of work piece 1 and 2 should be strictly met with credibility $99 \%$, while work piece 3 is more flexible with a $50 \%$ credibility to meet the delivery time. So, there are two priorities of scheduling. Three work pieces have fixed process route, each operation has many devices available for processing which differ in time and cost. According to experience and 
historical statistically analysis, the time and cost spent on each machine for one operation are stochastic variables. The time comply with exponential distribution while cost obeys normal distribution, whose parameters are shown in table 1 . Take " $R_{2} / 20 /(18,6)$ " as an example, it means work piece being processed on machine $\mathrm{R}_{2}$ with processing time obeying exponential distribution $\exp (20)$, cost obeying normal distribution $\mathrm{N}(18,6)$. We need to schedule for the order and implement optimum scheduling.

\begin{tabular}{|c|c|c|c|c|}
\hline \multirow{2}{*}{$\begin{array}{l}\text { Work } \\
\text { piece }\end{array}$} & \multirow{2}{*}{ Operation } & \multicolumn{3}{|c|}{ Class of machine available } \\
\hline & & Resource1 & Resource2 & Resource3 \\
\hline \multirow{5}{*}{$\begin{array}{c}\text { Work } \\
\text { piece } 1\end{array}$} & Lathing & $R_{2} / 20 /(18,6)$ & $R_{3} / 25 /(17,5)$ & \\
\hline & Milling & $R_{7} / 35 /(39,1)$ & $R_{8} / 30 /(45,2)$ & $R_{10} / 40 /(37,1)$ \\
\hline & Surface process & $R_{30} / 100 /(125,8)$ & $R_{31} / 90 /(140,6)$ & $R_{32} / 110 /(120,8)$ \\
\hline & $\begin{array}{l}\text { Lineation,Drilling } \\
\text { Assembling,Adjustment }\end{array}$ & $R_{20} / 40 /(50,4)$ & $R_{22} / 42 /(55,3)$ & \\
\hline & Line incision & $R_{17} / 40 /(45,4)$ & $R_{18} / 45 /(49,3)$ & \\
\hline \multirow{5}{*}{$\begin{array}{c}\text { Work } \\
\text { piece } 2\end{array}$} & Lathing & $\mathrm{R} 2 / 25 /(19,2)$ & $R_{3} / 33 /(18,2)$ & $R_{5} / 30 /(18,1)$ \\
\hline & Drilling,Milling & $R_{8} / 40 /(55,4)$ & $R_{10} / 50 /(50,3)$ & \\
\hline & Surface process & $R_{30} / 70 /(85,8)$ & $R_{31} / 67 /(90,6)$ & \\
\hline & Grinding & $R_{25} / 50 /(60,6)$ & $R_{26} / 52 /(60,5)$ & \\
\hline & Repairing Assembling & $R_{20} / 100 /(110,10)$ & $R_{21} / 115 /(117,10)$ & $R_{22} / 110 /(121,9)$ \\
\hline \multirow{5}{*}{$\begin{array}{c}\text { Work } \\
\text { piece } 3\end{array}$} & Milling & $R_{8} / 60 /(102,5)$ & $R_{9} / 70 /(85,7)$ & $R_{11} / 55 /(127,9)$ \\
\hline & Surface process & $R_{31} / 50 /(93,6)$ & $R_{32} / 60 /(55,2)$ & $R_{33} / 58 /(60,2)$ \\
\hline & Electrode NC process & $R_{35} / 33 /(36,4)$ & $R_{30} / 30 /(42,3)$ & \\
\hline & Electric-charge Process & $R_{19} / 40 /(50,4)$ & $R_{16} / 39 /(60,4)$ & \\
\hline & Repairing , assembling & $R_{21} / 160 /(220,12)$ & $R_{22} / 150 /(240,10)$ & \\
\hline
\end{tabular}

Table 1. Operations and machines available for work pieces

First, present a stochastic programming model, believable probabilities are:

$$
\eta=\gamma=v=0.8, \alpha 1=0.99, \alpha 2=0.5
$$

Then use hybrid intelligent algorithm with weights of three objectives being $1 / 3$ respectively to solve the problem. Get 200 groups of data samples through stochastic simulation (each group of data should be acquired by 500 times simulation), train the neural network with these data (15 input units, 18 hidden layer units and 6 output units), and calculate the weight. Finally, using genetic algorithm to find the optimum solution with 15 bits code, cross rate 0.5 and mutation rate 0.01 . After 30 generations' evolution, the result of schedule is as follows:

Work piece $1(2,7,30,22,17)$, work piece $2(5,8,30,25,20)$, work piece $3(8,32,35,19,22)$. The numbers in the bracket represent the serial number of machine for each operation. According to experience, this schedule sequence is a approximate optimum solution which has satisfied the requirements of all the chance constraints. 


\section{Conclusions}

After analyzing the stochastic factors as time and cost in production process, we have proposed stochastic programming model based on chance constraints to describe the multipriorities Flexible Job-Shop Scheduling problem aiming at objectives of shorter production time, lower cost and equilibrium of schedule. The advantage of this model is that it reflects the stochastic condition in real job-shop and proposes a hybrid intelligent algorithm which syncretizes many critical techniques such as the stochastic simulation, neural network and genetic algorithm etc., finally, a case study shows that our study can be applied to FJSP to get a approximate optimum solution.

\section{References}

Baoding, L.; Ruiqing, Z. \& Gang, W. (2003). Uncertain programming and application. In Chinese. Tsinghua University Press, Beijing

Croee, F. D. \& Tadei, R. (1995). A genetic algorithm for the Job Shop problem. Computer Ops. Res.vol.22(1), pp.15-24.

Foo, Y. S. \& Takefuji, Y. (1998). Integer linear programming neural networks for job-shop scheduling. In: IEEE Int Conf on NNS, San Diego,pp.320-332

Hongsen, Y. (1994). Research and Application of FMS Modeling, Scheduling, Controlling and Simulating. In Chinese. Harbin Institute of Technology.

Juanqi, Y. (1998). Synthetically Description and development of FMS Scheduling Research. In Chinese. Journal of Shanghai Jiaotong University,vol.32 (5),pp.124--127

Law, M. (1986). Simulation series: Part I: Introducing simulation: A tool for analyzing complex systems. Ind. Eng., May,: pp.46-63.

Peigen, L. (1998). Manufacturing System Performance Analysis Modeling - Theory and Methods. In Chinese. Huazhong University of Science and Technology, Wuhan

Philips, T.; Revenderen, A. etc. (1987). Theory and Practice of Operational Research, China Commercial Press, pp. 282--346. Beijing

Shuxia, L. (2004). MES certain technologies research under complex information environment. In Chinese. [Doctoral dissertation]. Wuhan

Taillard, E. (1990). Some efficient heuristic methods for the flow shop sequencing problem. Euro Operation Res. vol. 47(1), pp. 65-74.

Van Laarhoven. (1992). Job shop scheduling by simulated annealing, Operations Research.vol.40, pp.113--125

Zhaoqiang, G. \& Yiren, Z. (2002). Study on Job Shop Fuzzy Scheduling Problem Based on Genetic Algorithm. In Chinese. CIMS, 8 (8),pp.616-620. 


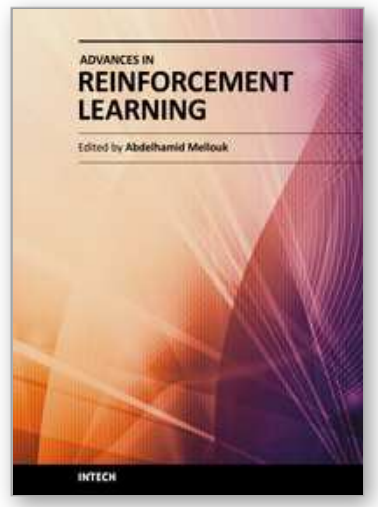

\author{
Advances in Reinforcement Learning \\ Edited by Prof. Abdelhamid Mellouk
}

ISBN 978-953-307-369-9

Hard cover, 470 pages

Publisher InTech

Published online 14, January, 2011

Published in print edition January, 2011

Reinforcement Learning $(R L)$ is a very dynamic area in terms of theory and application. This book brings together many different aspects of the current research on several fields associated to $R L$ which has been growing rapidly, producing a wide variety of learning algorithms for different applications. Based on 24 Chapters, it covers a very broad variety of topics in $R \mathrm{~L}$ and their application in autonomous systems. A set of chapters in this book provide a general overview of $R L$ while other chapters focus mostly on the applications of RL paradigms: Game Theory, Multi-Agent Theory, Robotic, Networking Technologies, Vehicular Navigation, Medicine and Industrial Logistic.

\title{
How to reference
}

In order to correctly reference this scholarly work, feel free to copy and paste the following:

Guojun Zhang, Haiping Zhu and Chaoyong Zhang (2011). Hybrid Intelligent Algorithm for Flexible Job-Shop Scheduling Problem under Uncertainty, Advances in Reinforcement Learning, Prof. Abdelhamid Mellouk (Ed.), ISBN: 978-953-307-369-9, InTech, Available from: http://www.intechopen.com/books/advances-inreinforcement-learning/hybrid-intelligent-algorithm-for-flexible-job-shop-scheduling-problem-under-uncertainty

\section{INTECH}

open science | open minds

\section{InTech Europe}

University Campus STeP Ri

Slavka Krautzeka 83/A

51000 Rijeka, Croatia

Phone: +385 (51) 770447

Fax: +385 (51) 686166

www.intechopen.com

\section{InTech China}

Unit 405, Office Block, Hotel Equatorial Shanghai

No.65, Yan An Road (West), Shanghai, 200040, China

中国上海市延安西路65号上海国际贵都大饭店办公楼 405 单元

Phone: +86-21-62489820

Fax: $+86-21-62489821$ 
(C) 2011 The Author(s). Licensee IntechOpen. This chapter is distributed under the terms of the Creative Commons Attribution-NonCommercialShareAlike-3.0 License, which permits use, distribution and reproduction for non-commercial purposes, provided the original is properly cited and derivative works building on this content are distributed under the same license. 